Supplementary Information

\title{
Enantioselective Intramolecular Alkene Hydroaminations Catalyzed by Yttrium Complexes of Axially Chiral Bis(thiolate) Ligands.
}

\author{
Joon Young Kim and Tom Livinghouse* \\ Department of Chemistry, Montana State University \\ Bozeman, MT 59717 \\ livinghouse@chemistry.montana.edu
}

Table of Contents

Experimental procedure and data of compounds: S2-S14

References: S15

Selected spectra: S16-S22

cf.) This is the 1st. version of using "track changes” function. 
General information: Melting points were obtained using a Mel-Temp II apparatus equipped with a digital thermometer and are uncorrected. Infrared spectra were recorded on a Perkin Elmer model 1600 FT-IR. Infrared spectra of solids were obtained by standard $\mathrm{KBr}$ pellet procedures.

${ }^{1} \mathrm{H}$ NMR spectra were recorded on a Bruker AVANCE DPX-300 (300MHz), or AVANCE DPX-500 (500MHz) spectrometer. Chemical shifts are reported in ppm from tetramethylsilane with the residual protic solvent resonance as the internal standard (chloroform: $\delta 7.24 \mathrm{ppm}$, or benzene: $\delta 7.22 \mathrm{ppm}$ ). Data are reported as follows: chemical shift, multiplicity $(\mathrm{s}=$ singlet, $\mathrm{d}=$ doublet, $\mathrm{t}=$ triplet, $\mathrm{q}=$ quartet, $\mathrm{m}=$ multiplets, br s = broad singlet, app. = apparent), integration, coupling constants (in $\mathrm{Hz}$ ), and assignments. ${ }^{13} \mathrm{C}$ NMR spectra were recorded on a Bruker AVANCE DPX-300 $(300 \mathrm{MHz})$, or AVANCE DPX-500 (500MHz) spectrometer with complete decoupling. Chemical shifts are reported in ppm from tetramethylsilane with the solvent as the internal standard $\left(\mathrm{CDCl}_{3}: \delta 77.0 \mathrm{ppm}\right)$. High Resolution Mass spectra were obtained on a Micromass LCT spectrometer, from the Mass Spectrometry and Proteomics Facility at the Ohio State University.

Analytical thin layer chromatography was performed on Polygram ${ }^{\circledR}$ SIL G/UV 254 $1.25 \mathrm{~mm}$ silica gel plates with a fluorescent indicator. Flash chromatography was performed on Merck silica gel 60. Solvents for extraction and flash chromatography were reagent grade.

All experiments were carried out under an argon atmosphere using standard anaerobic methods. Organoyttrium complexes were manipulated under an argon atmosphere in a glove box. Benzene- $\mathrm{D}_{6}$ and aminoalkenes were distilled from $\mathrm{Na}$ and $\mathrm{CaH}_{2}$ respectively, under an argon atmosphere, and stored at $-30{ }^{\circ} \mathrm{C}$ in a glove box. Aminoalkenes, pent-4-en-1-amine ${ }^{1}$, 2,2-dimethylpent-4-en-1-amine ${ }^{1}$, 2,2-dimethyl-5phenylpent-4-en-1-amine ${ }^{2}$, 2,2-dimethylhex-5-en-1-amine ${ }^{1}, \mathrm{~N}$-methylpent-4-en-1-amine ${ }^{3}$ were prepared according to reported procedures. 


\section{General Procedure for Intramolecular Alkene Hydroaminations}

In an argon-filled glove box, $\mathrm{Y}\left[\mathrm{N}(\mathrm{TMS})_{2}\right]_{3}$ (8) (9.1 $\left.\mathrm{mg}, 0.016 \mathrm{mmol}\right)$, the appropriate bis(thiol) proligand $7(0.016 \mathrm{mmol}), \mathrm{C}_{6} \mathrm{D}_{6}(0.7 \mathrm{~mL})$, and thiophene $(2.7 \mathrm{mg}$, $2.6 \mu \mathrm{L}, 0.032 \mathrm{mmol}$ ) were introduced sequentially into a J. Young NMR tube equipped with Teflon screw cap. The homogeneous reaction mixture was maintained at $60{ }^{\circ} \mathrm{C}$ or $75{ }^{\circ} \mathrm{C}$ respectively in a constant temperature oil bath until ligand attachment was judged complete by the disappearance of the $\mathrm{Y}\left[\mathrm{N}(\mathrm{TMS})_{2}\right]_{3}$ resonance in the ${ }^{1} \mathrm{H}$ NMR spectrum with concomitant production of (TMS $)_{2} \mathrm{NH}$. To the resulting complex was added the appropriate aminoalkene $10(0.32 \mathrm{mmol})$ and the reaction mixture was subsequently heated at $30{ }^{\circ} \mathrm{C}$ or $60{ }^{\circ} \mathrm{C}$ in an oil bath to achieve hydroamination. ${ }^{1} \mathrm{H}$ NMR spectroscopy using a pulse delay of $10 \mathrm{sec}$ was employed to monitor the progress of the reaction. The cyclic amine so produced was vacuum transferred from a J. Young NMR tube into a 25 $\mathrm{mL}$ round-bottomed flask which contained the appropriate amount of (R)-(-)-Oacetylmandelic acid [or (R)-(+)-2-methoxy-2-(trifluoromethyl)phenylacetic acid in the case of 11e] $(0.32 \mathrm{mmol})$ at $10^{-3}$ torr. This transfer was quantitated by washing the NMR tube with a small amount of $\mathrm{CDCl}_{3}$. The resulting mixture was stirred at $22{ }^{\circ} \mathrm{C}$ for $2-3 \mathrm{~h}$ and the volatiles were removed in vacuo. The resulting diastereomeric salt was then dissolved in $\mathrm{CDCl}_{3}$ and the enantiomeric excesses were determined by ${ }^{1} \mathrm{H} \mathrm{NMR}$ spectroscopy. The spectra of 11a/acetyl mandelic acid and 11e/acetylmandelic acid and those obtained from the enantioselective reactions are shown.

\section{Preparative Scale Hydroaminations}

\section{2,4,4-Trimethylpyrrolidine'HCl salt}

In an argon-filled glove box, Y[N(TMS) $)_{3}(8)(85.5 \mathrm{mg}, 0.15 \mathrm{mmol})$ and $N, N^{\prime}-$ bis(2-mercapto-5-methyl-3-dimethylphenylsilylbenzyl)- $N, N^{\prime}$-dimethyl-[1,1']-binaphthyl2,2'-diamine (7c) (130 mg, $0.15 \mathrm{mmol}$ ) were introduced into a $10 \mathrm{~mL}$ Schlenk flask equipped with a magnetic stirring bar and degassed benzene $(6.6 \mathrm{~mL})$ was subsequently added. The flask was sealed and the homogeneous reaction mixture was heated at $60{ }^{\circ} \mathrm{C}$ 
with stirring for $20 \mathrm{~h}$ to effect ligand attachment. The reaction mixture was cooled to room temperature and 2,2-dimethylpent-4-en-1-amine (10a) $(0.34 \mathrm{~g}, 0.45 \mathrm{~mL}, 3 \mathrm{mmol})$ was added via micro syringe. The reaction mixture was then heated to $60{ }^{\circ} \mathrm{C}$ in an oil bath with stirring for $9 \mathrm{~h}$ to complete hydroamination. The product amine and solvent were subsequently separated from the catalyst by vacuum transfer and the resultant mixture was cautiously added at $-15^{\circ} \mathrm{C}$ to a $6 \mathrm{M}$ solution of $\mathrm{HCl}$ in diethyl ether $(15 \mathrm{~mL})$. Removal of the solvents in vacuo provided the title compound $(0.45 \mathrm{~g}, 100 \%)$ as white solid.

${ }^{1} \mathrm{H} \mathrm{NMR} \mathrm{(CDCl} 3,500 \mathrm{MHz}$ ): $\delta$ ppm 10.0 (br s, $1 \mathrm{H},=\mathrm{NH}_{2}{ }^{+} \mathrm{Cl}^{-}$), 9.56 (br s, $1 \mathrm{H},=\mathrm{NH}_{2}{ }^{+} \mathrm{Cl}^{-}$), 3.88-3.86 (br m, $1 \mathrm{H},-\mathrm{CH}_{3}-\mathrm{CH}\left(\mathrm{CH}_{2}\right)-\mathrm{NH}_{2}{ }^{+} \mathrm{Cl}^{-}$), 3.13-3.12 (br m, $1 \mathrm{H},-\mathrm{CH}_{2}-\mathrm{NH}_{2}{ }^{+} \mathrm{Cl}^{-}$), 3.03-3.01 (br m, $1 \mathrm{H},-\mathrm{CH}_{2}-\mathrm{NH}_{2}{ }^{+} \mathrm{Cl}$ ), 1.97-1.94 (m, $\left.1 \mathrm{H},-\mathrm{CH}_{2}-\mathrm{CH}\left(\mathrm{CH}_{3}\right)-\right)$ ), 1.62-1.59 (m, $\left.1 \mathrm{H},-\mathrm{CH}_{2}-\mathrm{CH}\left(\mathrm{CH}_{3}\right)-\right), 1.56\left(\mathrm{~d}, 3 \mathrm{H},=\mathrm{CH}\left(\mathrm{CH}_{3}\right), J=6.5 \mathrm{~Hz}\right), 1.23\left(\mathrm{~s}, 3 \mathrm{H},=\mathrm{C}\left(\mathrm{CH}_{3}\right)_{2}\right), 1.19$ (s, $\left.3 \mathrm{H},=\mathrm{C}\left(\mathrm{CH}_{3}\right)_{2}\right)$.

${ }^{13} \mathrm{C}$ NMR (CDCl, $\left.500 \mathrm{MHz}\right): \delta$ ppm 56.6, 55.6, 47.3, 39.1, 27.5, 18.5.

\section{2-Benzyl-4,4-dimethylpyrrolidine'HCI salt}

In an argon-filled glove box, $\mathrm{Y}\left[\mathrm{N}(\mathrm{TMS})_{2}\right]_{3}(\mathbf{8})(85.5 \mathrm{mg}, 0.15 \mathrm{mmol})$ and $N, N^{\prime}-$ bis(2-mercapto-5-methyl-3-dimethylphenylsilylbenzyl)- $N, N^{\prime}$-dimethyl-[1,1']-binaphthyl2,2'-diamine (7c) (130 mg, $0.15 \mathrm{mmol})$ were introduced into a $10 \mathrm{~mL}$ Schlenk flask equipped with a magnetic stirring bar and degassed benzene $(6.6 \mathrm{~mL})$ was subsequently added. The flask was sealed and the homogeneous reaction mixture was heated at $60{ }^{\circ} \mathrm{C}$ with stirring for $10 \mathrm{~h}$ to effect ligand attachment. The reaction mixture was cooled to room temperature and 2,2-dimethyl-5-phenylpent-4-en-1-amine (10d) $(0.57 \mathrm{~g}, 3 \mathrm{mmol})$ was added via micro syringe. The reaction mixture was then heated to $60{ }^{\circ} \mathrm{C}$ in an oil bath with stirring for $3 \mathrm{~h}$ to complete hydroamination. The product amine and solvent were subsequently separated from the catalyst by vacuum transfer and the resultant mixture was cautiously added at $0{ }^{\circ} \mathrm{C}$ to a solution of $\mathrm{HCl}$ in $\mathrm{MeOH}(9 \mathrm{~mL})$ that was prepared by methanolysis of acetyl chloride $(0.6 \mathrm{~mL})$. Removal of the solvents in vacuo provided the title compound ( $0.7 \mathrm{~g}, 98 \%$ ) as white solid. 
${ }^{1} \mathrm{H}$ NMR (CDCl, $500 \mathrm{MHz}$ ): $\delta$ ppm 10.8 (br s, $1 \mathrm{H},=\mathrm{NH}_{2}{ }^{+} \mathrm{Cl}^{-}$), 9.75 (br s, $1 \mathrm{H},=\mathrm{NH}_{2}{ }^{+} \mathrm{Cl}^{-}$), 7.36-7.32 (m, 2H, -Ph), 7.24-7.19 (m, 3H, -Ph), 6.54 (d, 1H, =CHPh, J = 15.6 Hz), 6.22 (dm, 1H, =CHCH, $J=15.6 \mathrm{~Hz}), 3.91-3.88\left(\right.$ br m, $\left.1 \mathrm{H},=\mathrm{CH}-\mathrm{CH}\left(\mathrm{CH}_{2}\right)-\mathrm{NH}_{2}{ }^{+} \mathrm{Cl}^{-}\right), 3.11-3.08$ (br m, $1 \mathrm{H},-\mathrm{CH}_{2}-\mathrm{NH}_{2}{ }^{+} \mathrm{Cl}^{-}$), 3.07-2.98 (br m, $1 \mathrm{H},-\mathrm{CH}_{2}-\mathrm{NH}_{2}{ }^{+} \mathrm{Cl}^{-}$), 1.94-1.89 (m, $1 \mathrm{H},-\mathrm{CH}_{2}-$ $\left.\mathrm{CH}\left(\mathrm{CH}_{3}\right)^{-}\right)$, 1.59-1.54 (m, 1H, $\left.-\mathrm{CH}_{2}-\mathrm{CH}\left(\mathrm{CH}_{3}\right)^{-}\right), 1.22$ (s, 3H, =C( $\left.\left.\mathrm{CH}_{3}\right)_{2}\right), 1.19$ (s, 3H, $\left.=\mathrm{C}\left(\mathrm{CH}_{3}\right)_{2}\right)$.

${ }^{13} \mathrm{C} \mathrm{NMR}\left(\mathrm{CDCl}_{3}, 500 \mathrm{MHz}\right): \delta \mathrm{ppm}$ 138.9, 138.2, 135.4, 132.2, 132.1, 128.9, 57.6, 56.7, 47.3, 39.2, 18.4, 18.3 .

\section{Syntheses of BINAP-derived Proligands}

\section{2-Trimethylsilyl-4-methylbenzenethiol (3a)}

To a stirred solution of 4-methylbenzenethiol (6.2 g, $50 \mathrm{mmol})$ in cyclohexane (150 mL) was added $n$-BuLi (2.68 M in hexane, $41 \mathrm{~mL}, 0.11 \mathrm{mmol}$ ) dropwise with cooling in an ice-EtOH bath. TMEDA (12.8 g, $16 \mathrm{~mL}, 110 \mathrm{mmol})$ was then added to the reaction mixture with stirring at $-15{ }^{\circ} \mathrm{C}$. The reaction mixture was allowed to warm to ambient temperature and was stirred for $24 \mathrm{~h}$. The reaction mixture was cooled to $-15{ }^{\circ} \mathrm{C}$ and chlorotrimethylsilane (13.6 g, $18 \mathrm{~mL}, 125 \mathrm{mmol})$ was slowly added. The mixture was then warmed to ambient temperature and stirred for $24 \mathrm{~h}$. $10 \%$ Aqueous $\mathrm{HCl}$ solution was added and the aqueous layer was extracted with diethyl ether (3 x $75 \mathrm{~mL})$. The combined organic layers were dried over $\mathrm{MgSO}_{4}$. The suspension was filtered and the solvents were evaporated to obtain the crude bis(trimethylsilyl) derivative. This was dissolved in $\mathrm{MeOH}(100 \mathrm{~mL})$ and the mixture was heated at reflux for $18 \mathrm{~h}$. After the reaction mixture was cooled to room temperature, $2 \mathrm{~N} \mathrm{HCl} \mathrm{(25} \mathrm{mL})$ was added and the aqueous layer was extracted with diethyl ether $(3 \times 50 \mathrm{~mL})$. The combined organic phases were dried over $\mathrm{MgSO}_{4}$. The suspension was filtered and the ethereal layer was concentrated to obtain the crude product. This was purified by vacuum distillation (bp = 95-99 ${ }^{\circ} \mathrm{C}$ at aspirator pressure) to produce $3 \mathbf{a}$ as a colorless oil (7.4 g, 76\%).

FT-IR (KBr pellet) : 3245, 3220, 3070, 1605, 1555, 1450, 1320, 890, 770, $715 \mathrm{~cm}^{-1}$. 
${ }^{1} \mathrm{H}$ NMR (CDCl, $\left.500 \mathrm{MHz}\right): \delta$ ppm 7.24-7.21 (m, 2H, $\mathrm{PhH}$ ), 7.03 (d, 1H, $J=9 \mathrm{~Hz}, \mathrm{Ph} H$ ), 3.43 (s, 1H, -SH), 2.3 (s, 3H, Ph-Me), 0.39 (s, 9H, $\mathrm{SiMe}_{3}$ ).

${ }^{13} \mathrm{C}$ NMR (CDCl $\left.3,500 \mathrm{MHz}\right): \delta$ ppm 140.0, 135.9, 134.9, 133.0, 132.1, 130.5, 21.0, -0.3.

\section{2-Triethylsilyl-4-methylbenzenethiol (3b)}

This compound was prepared in a fashion analogous to 3a, but utilizing chlorotriethylsilane. The product was purified by vacuum distillation (bp $=99-105{ }^{\circ} \mathrm{C}$ in aspirator pressure) to provide $6.8 \mathrm{~g}$ (82\%).

FT-IR (KBr pellet) : 3350, 3345, 3165, 1710, 1665, 1350, 1315, 880, 780, $725 \mathrm{~cm}^{-1}$.

${ }^{1} \mathrm{H}$ NMR (CDCl $3,500 \mathrm{MHz}$ ): $\delta$ ppm 7.25-7.22 (m, 2H, $\mathrm{PhH}$ ), 7.02 (d, $1 \mathrm{H}, J=9 \mathrm{~Hz}, \mathrm{Ph} H$ ), 6.81 (s, 1H, -SH), 2.35 (s, 3H, Ph-Me), 0.96 (t, 9H, $J=7.5 \mathrm{~Hz}, \mathrm{SiCH}_{2} \mathrm{CH}_{3}$ ) 0.48 (q, 6H, $J$ $\left.=7 \mathrm{~Hz}, \mathrm{SiCH}_{2} \mathrm{CH}_{3}\right)$.

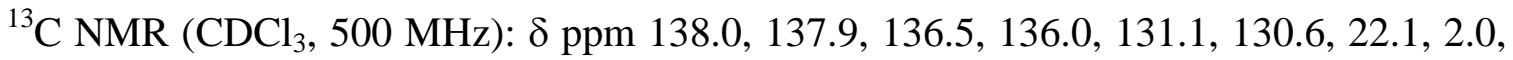
1.2 .

\section{2-Dimethylphenylsilyl-4-methylbenzenethiol (3c)}

This compound was prepared in a fashion analogous to 3a, but utilizing chlorodimethylphenylsilane to provide $5.9 \mathrm{~g}$ (82\%) of 3c.

FT-IR (KBr pellet) : 3235, 3210, 3040, 1605, 1585, 1575, 1555, 1450, 1320, 880, 770 $\mathrm{cm}^{-1}$.

${ }^{1} \mathrm{H}$ NMR ( $\left.\mathrm{CDCl}_{3}, 500 \mathrm{MHz}\right): \delta \mathrm{ppm} 8.1$ (d, 2H, $\left.J=9 \mathrm{~Hz}, \mathrm{SiMe}_{2} \mathrm{PhH}\right), 7.86(\mathrm{~m}, 3 \mathrm{H}$, $\mathrm{SiMe}_{2} \mathrm{Ph} H$ ), 7.23-7.20 (m, 2H, $\mathrm{Ph} H$ ), 7.0 (d, 1H, $J=9 \mathrm{~Hz}, \mathrm{Ph} H$ ), 3.43 (s, $1 \mathrm{H},-\mathrm{SH}$ ), 2.35 (s, 3H, Ph-Me), 0.33 (s, 6H, SiMe $2 \mathrm{Ph}$ ).

${ }^{13} \mathrm{C} \mathrm{NMR}\left(\mathrm{CDCl}_{3}, 500 \mathrm{MHz}\right): \delta$ ppm 140.1, 138.2, 136.2 135.1, 132.1, 130.1, 129.2, 24.5, -2.1 .

\section{2-Mercapto-5-methyl-3-trimethylsilylbenzoic acid (4a)}

A $50 \mathrm{~mL}$ argon-flushed flask equipped with a magnetic stirring bar and argon inlet was charged with $3 a$ ( $1.96 \mathrm{~g}, 10 \mathrm{mmol})$ and TMEDA (2.8 g, $3.7 \mathrm{~mL}, 24 \mathrm{mmol})$. The reaction mixture was stirred and cooled to $-15{ }^{\circ} \mathrm{C}$ and then $n$-BuLi (2.5 M in hexane, 9.2 
$\mathrm{mL}, 24 \mathrm{mmol}$ ) was added dropwise. The reaction mixture, which gradually became homogeneous, was then stirred for $24 \mathrm{~h}$ at ambient temperature. The mixture was diluted with cyclohexane $(20 \mathrm{~mL})$ and this solution was added dropwise via cannula to a $100 \mathrm{~mL}$ flask that contained methyl chloroformate $(2.27 \mathrm{~g}, 1.9 \mathrm{~mL}, 24 \mathrm{mmol})$ cooled in an iceEtOH bath. The reaction mixture was allowed to warm to room temperature and then stirred overnight (18 h). Water was added, and the separated aqueous layer was extracted three times with diethyl ether. The combined organic layers were dried over $\mathrm{MgSO}_{4}$, and the filtrate was concentrated to provide a crude solid. To a solution of $\mathrm{LiOH}(1.1 \mathrm{~g}, 44$ $\mathrm{mmol})$ and $\mathrm{MeOH}(20 \mathrm{~mL})$ was added a solution of the resulting solid in $\mathrm{MeOH}(15 \mathrm{~mL})$ at $-15{ }^{\circ} \mathrm{C}$. After the addition was completed, the mixture was allowed to warm to room temperature and stirred for $12 \mathrm{~h}$. $10 \% \mathrm{HCl}$ was added and the aqueous layer was extracted with diethyl ether ( $3 \times 70 \mathrm{~mL})$. The combined organic layers were dried over $\mathrm{MgSO}_{4}$. The filtrate was concentrated to provide the crude solid product. The product was purified by recrystallization from toluene to produce $\mathbf{4 a}$ as a yellow solid, $\mathrm{mp}=148$ $151{ }^{\circ} \mathrm{C}(2.18 \mathrm{~g}, 91 \%)$.

FT-IR (KBr pellet ): 3580, 3100, 3070, 2940, 1750, 1615, 1540, 1460, 1270, 990, 870, $815 \mathrm{~cm}^{-1}$.

${ }^{1} \mathrm{H}$ NMR (CDCl $3,500 \mathrm{MHz}$ ): $\delta$ ppm 7.89 (s, 1H, $\mathrm{Ph} H$ ), 7.41 (s, 1H, PhH), 2.95 (br s, 2H, -SH, -COOH), 2.31 (s, 3H, $\mathrm{PhMe}$ ), 0.36 (s, 9H, $\mathrm{SiMe}_{3}$ ).

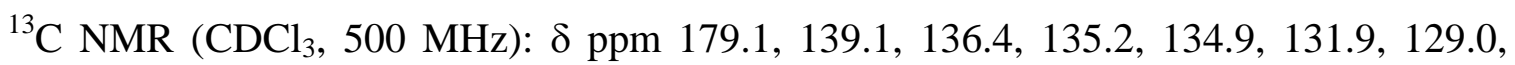
24.3, -0.3 .

\section{2-Mercapto-5-methyl-3-triethylsilylbenzoic acid (4b)}

This compound was prepared in a fashion analogous to $\mathbf{4 a}$ to provide $\mathbf{4 b}, \mathrm{mp}=$ $141-146{ }^{\circ} \mathrm{C}(3.1 \mathrm{~g}, 90 \%)$.

FT-IR (KBr pellet) : 3585, 3205, 3165, 2995, 1740, 1625, 1550, 1465, 990, 870, $815 \mathrm{~cm}^{-1}$. ${ }^{1} \mathrm{H}$ NMR ( $\mathrm{CDCl}_{3}, 500 \mathrm{MHz}$ ): $\delta$ ppm 10.8 (br s, $1 \mathrm{H},-\mathrm{COOH}$ ), 7.85 (s, 1H, $\mathrm{Ph} H$ ), 7.49 (s, 1H, $\mathrm{PhH}$ ), 6.6 (s, $1 \mathrm{H},-\mathrm{SH}$ ), 0.95 (t, 9H, $J=7.5 \mathrm{~Hz}, \mathrm{SiCH}_{2} \mathrm{CH}_{3}$ ) 0.45 (q, $6 \mathrm{H}, J=7 \mathrm{~Hz}$, $\left.\mathrm{SiCH}_{2} \mathrm{CH}_{3}\right)$. 
${ }^{13} \mathrm{C}$ NMR $\left(\mathrm{CDCl}_{3}, 500 \mathrm{MHz}\right): \delta \mathrm{ppm} 175.5,139.6,136.4,135.1,133.9,131.4,130.3$, 25.3, 2.1, 1.5 .

\section{2-Mercapto-5-methyl-3-dimethylphenylsilylbenzoic acid (4c)}

This compound was prepared according to the procedure described for $4 \mathbf{a}$ to provide 4c (mp = 161-163 $\left.{ }^{\circ} \mathrm{C}\right), 2.7 \mathrm{~g}(90 \%)$.

FT-IR (KBr pellet) : 3555, 3215, 3135, 2985, 1695, 1585, 1570, 1560, 1450, 990, 870, $815 \mathrm{~cm}^{-1}$.

${ }^{1} \mathrm{H}$ NMR ( $\left.\mathrm{CDCl}_{3}, 500 \mathrm{MHz}\right): \delta$ ppm 11.0 (s, 1H, -COOH), 7.99 (s, 1H, PhH), 7.54 (m, 2H, $\mathrm{SiMe}_{2} \mathrm{PhH}$ ), 7.41-7.36 (m, 2H, SiMe $2 \mathrm{PhH}, \mathrm{PhH}$ ), 3.12 (s, 1H, -SH), 2.35 (s, 3H, $\mathrm{PhMe}$ ), 0.36 (s, 6H, SiMe $2 \mathrm{Ph})$.

${ }^{13} \mathrm{C} \mathrm{NMR}\left(\mathrm{CDCl}_{3}, 500 \mathrm{MHz}\right): \delta \mathrm{ppm} 178.8,148.8,142.2,139.2,139.7,138.2,136.4$, 135.2, 133.3, 130.1, 25.3, -2.0.

\section{N,N'-Bis(2-mercapto-5-methyl-3-trimethylsilylphenyl)-[1,1']-binaphthyl-2,2'- diamide (6a)}

A solution of 4a (1.3 g, $5.5 \mathrm{mmol})$ and triphenyl phosphite $(1.5 \mathrm{~mL}, 5.6 \mathrm{mmol})$ in pyridine $(50 \mathrm{~mL})$ was heated at $70{ }^{\circ} \mathrm{C}$ for $3 \mathrm{~h}$. To this mixture was added a solution of (R)-(+)-1,1'-binaphthyl-2,2'-diamine (0.7 mg, $2.5 \mathrm{mmol})$ in pyridine (2 mL). The reaction mixture was subsequently heated at $100{ }^{\circ} \mathrm{C}$ for $18 \mathrm{~h}$. After the mixture was cooled to room temperature, $5 \%$ aqueous $\mathrm{HCl}(5 \mathrm{~mL})$ and water $(3 \mathrm{~mL})$ were added in sequence. The aqueous layer was extracted with ethyl acetate, the combined organic layers were washed with saturated $\mathrm{NH}_{4} \mathrm{Cl}$, saturated $\mathrm{NaHCO}_{3}$ and saturated $\mathrm{NaCl}$ solutions in this order, and the organic phase was dried over $\mathrm{MgSO}_{4}$. The suspension was filtered and the filtrate was concentrated to provide the crude product, which was purified by column chromatography (hexane : ethyl acetate $=4: 1$ to $2: 1$ gradient) to produce 6a, $\mathrm{mp}=306{ }^{\circ} \mathrm{C}$ (dec.) (3.2 g, 80\%).

FT-IR (KBr pellet) : 3570, 3210, 3090, 2940, 1710, 1615, 1580, 1540, 1460, 1270, 995, $860,805 \mathrm{~cm}^{-1}$. 
${ }^{1} \mathrm{H}$ NMR $\left(\mathrm{CDCl}_{3}, 500 \mathrm{MHz}\right): \delta$ ppm 10.1 (s, 2H, 2NH), 8.03 (d, 2H, binaphthyl-H, $J=$ 9Hz), 7.93 (m, 3H, binaphthyl- $H, \mathrm{Ph} H), 7.44$ (m, 3H, binaphthyl- $H, \mathrm{Ph} H)$, 7.32 (m, 2H, binaphthyl- $H$ ), 7.21 (m, 2H, binaphthyl- $H$ ), 7.05 (d, 2H, binaphthyl- $H, J=9 H z), 2.35$ (s, 6H, $\mathrm{PhMe}$ ), 0.38 (s, 18H, $\left.\mathrm{SiMe}_{3}\right)$.

${ }^{13} \mathrm{C} \mathrm{NMR}\left(\mathrm{CDCl}_{3}, 500 \mathrm{MHz}\right): \delta \mathrm{ppm} 177.1,138.1,136.2,134.5,133.4,132.1,131.0$, $129.7,128.3,127.4,125.4,121.2,120.2,118.2,24.5,-0.7$.

\section{$N, N^{\prime}$-Bis(2-mercapto-5-methyl-3-triethylsilylphenyl)-[1,1']-binaphthyl-2,2'-diamide}

(6b)

This compound was prepared in a fashion analogous to 6a to provide $2.0 \mathrm{~g}$ (82\%) of $\mathbf{6 b}, \mathrm{mp}=295^{\circ} \mathrm{C}$ (dec.).

FT-IR (KBr pellet) : 3560, 3200, 3085, 2940, 1710, 1615, 1580, 1540, 1460, 1270, 995, $860,810 \mathrm{~cm}^{-1}$.

${ }^{1} \mathrm{H}$ NMR $\left(\mathrm{CDCl}_{3}, 500 \mathrm{MHz}\right): \delta$ ppm 10.7. (s, 2H, 2NH), 8.0 (d, 2H, binaphthyl-H, $J=$ 9Hz), 7.89 (m, 3H, binaphthyl-H, $\mathrm{Ph} H$ ), 7.46 (m, 3H, binaphthyl- $H, \mathrm{Ph} H), 7.3$ (m, 2H, binaphthyl-H), 7.19 (m, 2H, binaphthyl- $H$ ), 7.0 (d, 2H, binaphthyl- $H, J=9 \mathrm{~Hz}$ ), 6.8 (s, 1H, -SH), 2.33 (s, 6H, PhMe), 0.96 (t, 9H, $\left.J=7.5 \mathrm{~Hz}, \mathrm{SiCH}_{2} \mathrm{CH}_{3}\right) 0.48$ (q, 6H, $J=7 \mathrm{~Hz}$, $\left.\mathrm{SiCH}_{2} \mathrm{CH}_{3}\right)$.

${ }^{13} \mathrm{C}$ NMR $\left(\mathrm{CDCl}_{3}, 500 \mathrm{MHz}\right): \delta \mathrm{ppm} 179.1,139.1,135.3,134.0,133.1,131.0,129.6$, 128.2, 127.6, 125.0, 120.3, 120.2, 118.2, 24.5, 2.2, 1.6 .

\section{N,N'-Bis(2-mercapto-5-methyl-3-dimethylphenylsilylphenyl)-[1,1']-binaphthyl-2,2'- diamide (6c)}

This compound was prepared as described for $\mathbf{6 a}$ to provide $2.5 \mathrm{~g}(90 \%)$ of $\mathbf{6 c}$, $\mathrm{mp}=311^{\circ} \mathrm{C}($ dec. $)$.

FT-IR (KBr pellet) : 3600, 3300, 3095, 2950, 1710, 1615, 1585, 1540, 1360, 1270, 995, $860,810 \mathrm{~cm}^{-1}$.

${ }^{1} \mathrm{H}$ NMR (CDCl, $\left.500 \mathrm{MHz}\right): \delta$ ppm 10.3 (s, 2H, 2NH), 8.53 (m, 2H, binaphthyl-H), 7.93 (m, 4H, binaphthyl- $H$, $\mathrm{Ph} H$ ), 7.61-7.56 (m, 6H, binaphthyl- $H, \mathrm{SiMe}_{2} \mathrm{Ph} H$ ), 7.50-7.44 (m, 8H, binaphthyl- $H, \mathrm{Ph} H, \mathrm{SiMe}_{2} \mathrm{Ph} H$ ), 7.32 (m, 2H, binaphthyl-H), 7.21 (m, 2H, 
binaphthyl- $H$ ), 7.05 (m, 2H, binaphthyl-H), 3.35 (s, 2H, -SH), 2.35 (s, 6H, PhMe), 0.36 (s, $\left.6 \mathrm{H}, \mathrm{SiMe}_{2} \mathrm{Ph}\right)$.

${ }^{13} \mathrm{C}$ NMR (CDCl $\left.3,500 \mathrm{MHz}\right): \delta \mathrm{ppm}$ 176.1, 140.1, 138.9, 138.1, 137.2, 136.2, 136.1, 135.8, 135.0, 134.4, 134.3, 134.1, 133.1, 132.9, 132.6, 130.8, 130.7, 120.1, 25.5, -1.0.

\section{$N, N^{\prime}$-Bis(2-mercapto-5-methyl-3-trimethylsilylbenzyl)- $N, N^{\prime}$-dimethyl-[1,1']- binaphthyl-2,2'-diamine (7a)}

To a stirred suspension of $\mathrm{LiAlH}_{4}(0.38 \mathrm{~g}, 10 \mathrm{mmol})$ in toluene $(15 \mathrm{~mL})$ was slowly added 1-methylpyrrolidine $(10.1 \mathrm{~mL}, 100 \mathrm{mmol})$ at room temperature. The mixture was stirred for $26 \mathrm{~h}$ at $25{ }^{\circ} \mathrm{C}$ and the stirring was then stopped to precipitate the solids. ${ }^{4}$ The supernatant solution, which contains $\mathrm{AlH}_{3} \cdot\left(\mathrm{CH}_{2}\right)_{4} \mathrm{~N}_{-}-\mathrm{CH}_{3}$ was carefully transferred to a solution of $\mathbf{6 a}(0.2 \mathrm{~g}, 0.27 \mathrm{mmol})$ in THF via cannula with ice-EtOH cooling. After the addition was complete, the reaction mixture was stirred for 3.5 days at $25{ }^{\circ} \mathrm{C}$. Water $(15 \mathrm{~mL})$ was added to the reaction mixture at $0{ }^{\circ} \mathrm{C}$. The aqueous layer was extracted with diethyl ether ( 3 x $25 \mathrm{~mL}$ ) and the combined organic layers were dried over $\mathrm{MgSO}_{4}$. The filtrate was concentrated and the residue was dissolved in toluene (2.5 mL). The solution was filtered through a short plug of silica gel and concentrated in vacuo. The solid was mixed with a suspension of $\mathrm{NaBH}_{4}(0.12 \mathrm{~g}, 3 \mathrm{mmol})$ in THF and the resulting mixture was added dropwise to a solution of $37 \%$ formaldehyde $(1 \mathrm{~mL}, 10$ mmol) and $20 \%$ aqueous $\mathrm{H}_{2} \mathrm{SO}_{4}(1 \mathrm{~mL})$ in THF (5 mL) very slowly (30 min-60 min) at room temperature. The mixture was stirred for additional $60 \mathrm{~min}$ and then poured into $2 \%$ aqueous $\mathrm{KOH}(100 \mathrm{~mL}) .{ }^{5}$ The resulting suspension was extracted with ethyl acetate to obtain a total volume of $250 \mathrm{~mL}$ and the organic layer was dried over $\mathrm{MgSO}_{4}$, filtered and evaporated. The crude product was purified by recrystallization (methylcyclohexane $/$ toluene $=1 / 2)$ to give $7 \mathrm{a}, \mathrm{mp}=251-254{ }^{\circ} \mathrm{C}(0.13 \mathrm{~g}, 64 \%)$.

FT-IR (KBr pellet) : 3090, 2940, 1710, 1615, 1580, 1540, 1460, 1270, 995, 860, $800 \mathrm{~cm}^{-1}$. ${ }^{1} \mathrm{H}$ NMR ( $\left.\mathrm{CDCl}_{3}, 500 \mathrm{MHz}\right): \delta$ ppm 8.56 (d, 2H, binaphthyl- $\left.H, J=9 \mathrm{~Hz}\right), 8.1(\mathrm{~d}, 2 \mathrm{H}$, binaphthyl- $H, J=9 \mathrm{~Hz}), 7.89$ (m, 2H, binaphthyl- $H$ ), 7.47 (m, 2H, binaphthyl- $H, \mathrm{Ph} H$ ), 7.44 (m, 2H, binaphthyl- $H$ ), 7.2 (d, 2H, binaphthyl- $H, J=9 H z), 7.05$ (m, 4H, binaphthyl- 
H, $\mathrm{PhH}$ ), 6.11 (s, 2H, -SH), 4.28-4.35 (m, 4H, $\mathrm{CH}_{2} \mathrm{~N}$ ), 2.35 (s, 6H, PhMe), 0.36 (s, 18H, $\left.\mathrm{SiMe}_{3}\right)$.

${ }^{13} \mathrm{C}$ NMR (CDCl $\left.3500 \mathrm{MHz}\right): \delta \mathrm{ppm} 146.2,138.5,137.8,134.5,133.0,131.1,130.0$, 128.1, 126.4, 128.5, 122.8, 121.5, 120.3, 43.1, 25.1, -0.3.

HRMS : Observed $\left[\left\{\mathrm{M}-\left(\mathrm{Me}_{3} \mathrm{Si}\right)\right\}+\mathrm{Na}\right]=584.1593$, Calculated $\left[\left\{\mathrm{M}-\left(\mathrm{Me}_{3} \mathrm{Si}\right)\right\}+\mathrm{Na}=\right.$ 584.1592.

\section{$N, N^{\prime}$-Bis(2-mercapto-5-methyl-3-triethylsilylbenzyl)- $N, N^{\prime}$-dimethyl-[1,1']-}

\section{binaphthyl-2,2'-diamine (7b)}

This compound was prepared in a fashion analogous to $\mathbf{7 a}$ to provide $\mathbf{7 b}(\mathrm{mp}=$ 249-251 $\left.{ }^{\circ} \mathrm{C}\right), 0.11 \mathrm{~g}(61 \%)$.

FT-IR (KBr pellet) : 3090, 2940, 16200, 1615, 1580, 1540, 1460, 1270, 995, 860, 800 $\mathrm{cm}^{-1}$.

${ }^{1} \mathrm{H}$ NMR $\left(\mathrm{CDCl}_{3}, 500 \mathrm{MHz}\right): \delta$ ppm 8.58 (d, 2H, binaphthyl- $\left.H, J=9 \mathrm{~Hz}\right), 8.22$ (d, 2H, binaphthyl- $H, J=9 \mathrm{~Hz}$ ), 7.86 (m, 2H, binaphthyl- $H$ ), 7.51 (m, 2H, binaphthyl- $H, \mathrm{Ph}$ ), 7.44 (m, 2H, binaphthyl- $H$ ), 7.2 (d, 2H, binaphthyl- $H, J=9 \mathrm{~Hz}$ ), 6.99 (m, 4H, binaphthyl$H, \mathrm{Ph}), 6.23$ (s, 2H, -SH), 4.28-4.35 (m, 4H, $\left.\mathrm{CH}_{2} \mathrm{~N}\right), 2.35$ (s, 6H, PhMe), 0.96 (t, 9H, $J=$ $7.5 \mathrm{~Hz}, \mathrm{SiCH}_{2} \mathrm{CH}_{3}$ ) 0.48 (q, $6 \mathrm{H}, J=7 \mathrm{~Hz}, \mathrm{SiCH}_{2} \mathrm{CH}_{3}$ ).

${ }^{13} \mathrm{C}$ NMR $\left(\mathrm{CDCl}_{3}, 500 \mathrm{MHz}\right): \delta$ ppm 146.8, 138.5, 137.6, 134.5, 134.1, 130.7, 130.1, 129.3, 128.5, 122.0, 120.5, 120.2, 43.0, 25.7, 3.1, 2.3.

HRMS : Observed $\left[\left\{\mathrm{M}-\left(\mathrm{Et}_{3} \mathrm{Si}\right)\right\}+\mathrm{Na}\right]=584.1590$, Calculated $\left[\left\{\mathrm{M}-\left(\mathrm{Et}_{3} \mathrm{Si}\right)\right\}+\mathrm{Na}\right]=$ 584.1592.

\section{$N, N$ '-Bis(2-mercapto-5-methyl-3-dimethylphenylsilylbenzyl)- $N, N^{\prime}$-dimethyl-[1,1']- binaphthyl-2,2'-diamine (7c)}

This compound was prepared as described for $7 \mathbf{a}$ to provide $7 \mathbf{c}\left(\mathrm{mp}=273-278{ }^{\circ} \mathrm{C}\right)$, $0.08 \mathrm{~g}(69 \%)$.

FT-IR (KBr pellet) : 3075, 2950, 1630, 1615, 1580, 1540, 1460, 1270, 995, 860, $810 \mathrm{~cm}^{-1}$. ${ }^{1} \mathrm{H}$ NMR (CDCl, $500 \mathrm{MHz}$ ): $\delta$ ppm 8.56 (m, 2H, binaphthyl- $H, J=9 \mathrm{~Hz}$ ), 8.1-7.93 (m, 4H, binaphthyl- $H$, $\mathrm{Ph} H$ ), 7.76-7.59 (m, 6H, binaphthyl- $H$, $\mathrm{SiMe}_{2} \mathrm{Ph} H$ ), 7.50-7.44 (m, 8H, 
binaphthyl- $H, \mathrm{Ph} H, \mathrm{SiMe}_{2} \mathrm{Ph} H$ ), 7.34 (m, 2H, binaphthyl- $H$ ), 7.2 (d, 2H, binaphthyl- $H, J$ = 9Hz), $7.00(\mathrm{~m}, 2 \mathrm{H}$, binaphthyl- $H)$, 4.61-4.58 (m, 4H, $\left.\mathrm{CH}_{2} \mathrm{~N}\right), 3.36(\mathrm{~s}, 2 \mathrm{H},-\mathrm{SH}), 2.35(\mathrm{~s}$, 6H, $\mathrm{PhMe}$ ), 0.36 (s, 6H, $\mathrm{SiMe}_{3} \mathrm{Ph}$ ).

${ }^{13} \mathrm{C}$ NMR $\left(\mathrm{CDCl}_{3}, 500 \mathrm{MHz}\right): \delta \mathrm{ppm}$ 150.2, 138.6, 137.8, 137.5, 137.2, 136.8, 136.1, 135.9, 135.1, 134.5, 134.3, 134.0, 133.7, 133.1, 132.8, 131.2, 130.8, 130.7, 118.6, 50.1, 25.5, -1.0 .

HRMS : Observed $[\mathrm{M}+\mathrm{Na}]=875.3323$, Calculated $[\mathrm{M}+\mathrm{Na}]=875.3321$.

\section{Syntheses of Amino-alkenes}

\section{2,2-Dimethylpent-4-en-1-amine (10a)}

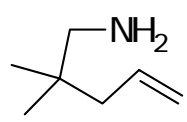

To a stirred suspension of $\mathrm{LiAlH}_{4}(2.66 \mathrm{~g}, 70 \mathrm{mmol})$ in dry $\mathrm{Et}_{2} \mathrm{O}(60 \mathrm{~mL})$ was added 2,2-dimethylpent-4-en-1-nitrile ( $3.8 \mathrm{~g}, 35 \mathrm{mmol})^{1}$ dropwise at room temperature. After heating at reflux overnight, the reaction mixture was cooled to $0{ }^{\circ} \mathrm{C}$ and carefully quenched via sequential addition of $\mathrm{H}_{2} \mathrm{O}(2 \mathrm{~mL}), 15 \%$ aqueous $\mathrm{NaOH}(2 \mathrm{~mL})$ and $\mathrm{H}_{2} \mathrm{O}$ (4 $\mathrm{mL}$ ). The mixture was subsequently stirred at room temperature for $2 \mathrm{~h}$ and anhydrous $\mathrm{MgSO}_{4}$ (4 g) was added. The suspension was filtered and extracted 3 times with diethyl ether $(15 \mathrm{~mL})$. The filtrate was concentrated in vacuo and the residue was distilled to afford $3.5 \mathrm{~g}$ (89 \%) of the title compound 10a $\left(\mathrm{bp}=51-52^{\circ} \mathrm{C}\right)$.

${ }^{1} \mathrm{H}$ NMR $\left(\mathrm{CDCl}_{3}, 500 \mathrm{MHz}\right): \delta \mathrm{ppm}$ 5.73-5.79 (m, $\left.1 \mathrm{H}, \mathrm{CH}=\mathrm{CH}_{2}\right)$, 4.98-5.08 (m, 2H, $\left.\mathrm{CH}=\mathrm{CH}_{2}\right), 2.45\left(\mathrm{~s}, 2 \mathrm{H}, \mathrm{NCH}_{2}\right), 1.99\left(\mathrm{~d}, 2 \mathrm{H}, \mathrm{CH}_{2} \mathrm{CH}=, J_{\mathrm{HH}}=7.6 \mathrm{~Hz}\right), 1.16($ br s, $2 \mathrm{H}$, $\mathrm{NH}_{2}$ ), 0.88 (s, 6H, $\mathrm{Me}_{2}$ ).

${ }^{13} \mathrm{C}$ NMR (CDCl $\left.3,500 \mathrm{MHz}\right): \delta$ ppm 135.9, 117.1, 53.1, 44.6, 36.1, 23.9. 


\section{Pent-4-en-1-amine (10b)}

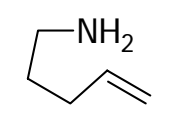

This compound was prepared in a fashion analogous to 10a, but utilizing pent-4en-1-nitrile. ${ }^{1}$ Product 10b (1.8 g, 81\%) was obtained by distillation (bp = 98-104 ${ }^{\circ} \mathrm{C}$ ).

${ }^{1} \mathrm{H}$ NMR $\left(\mathrm{CDCl}_{3}, 500 \mathrm{MHz}\right): \delta \mathrm{ppm}$ 5.71-5.78 (m, 1H, $\left.\mathrm{CH}=\mathrm{CH}_{2}\right)$, 5.01-5.05 (m, 2H, $\mathrm{CH}=\mathrm{CH}_{2}$ ), 2.71 (t, $2 \mathrm{H}, J=7 \mathrm{~Hz}, \mathrm{H}_{2} \mathrm{NCH}_{2}$ ), 2.10 (q, 2H, $J=7.2 \mathrm{~Hz}, \mathrm{CH}_{2} \mathrm{CH}_{=} \mathrm{CH}_{2}$ ), 1.511.57 (m, 2H, $\mathrm{CH}_{2} \mathrm{CH}_{2} \mathrm{CH}_{2}$ ), 1.21 (br s, $2 \mathrm{H}, \mathrm{NH}_{2}$ ).

${ }^{13} \mathrm{C} \mathrm{NMR}\left(\mathrm{CDCl}_{3}, 500 \mathrm{MHz}\right): \delta \mathrm{ppm} 137.1,115.9,43.2,35.5,31.0$.

\section{2,2-Dimethylhex-5-en-1-amine (10c)}

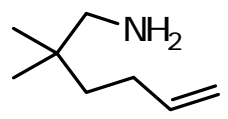

This compound was prepared as described for 10a, but utilizing 2,2-dimethylhex5-en-1-nitrile. ${ }^{1}$ Product was purified by vacuum distillation (bp $=70-74{ }^{\circ} \mathrm{C}$ at aspirator pressure) to provide $2.3 \mathrm{~g}$ of $\mathbf{1 0 c}(86 \%)$.

${ }^{1} \mathrm{H}$ NMR $\left(\mathrm{CDCl}_{3}, 500 \mathrm{MHz}\right): \delta \mathrm{ppm}$ 5.69-5.75 (m, 1H, $\left.\mathrm{CH}=\mathrm{CH}_{2}\right), 4.97-5.03(\mathrm{~m}, 2 \mathrm{H}$, $\left.\mathrm{CH}=\mathrm{CH}_{2}\right), 2.57\left(\mathrm{~s}, 2 \mathrm{H}, \mathrm{CH}_{2} \mathrm{~N}\right), 2.03-1.97\left(\mathrm{~m}, 2 \mathrm{H}, \mathrm{CH}_{2} \mathrm{CH}=\right), 1.22-1.26\left(\mathrm{~m}, 2 \mathrm{H}, \mathrm{CH}_{2}\right)$, $1.11\left(\mathrm{~s}, 6 \mathrm{H}, 2 \mathrm{CH}_{3}\right)$.

${ }^{13} \mathrm{C} \mathrm{NMR}\left(\mathrm{CDCl}_{3}, 500 \mathrm{MHz}\right): \delta \mathrm{ppm} 138.8,115.7,52.9,41,8,34.2,28.4,24.9$.

\section{2,2-Dimethyl-5-phenylpent-4-en-1-amine (10d)}

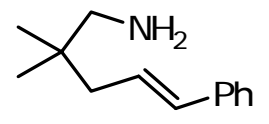

This compound was prepared according to the procedure as described for 10a, but utilizing 2,2-dimethyl-5-phenylpent-4-en-1-nitrile. ${ }^{2}$ The product was distilled (bp = 115$118^{\circ} \mathrm{C}$ ) to provide $1.8 \mathrm{~g}$ of $\mathbf{1 0 d}(93 \%)$. 
${ }^{1} \mathrm{H}$ NMR (CDCl, $\left.500 \mathrm{MHz}\right): \delta$ ppm 7.16-7.34 (m, 5H, Ph), 6.38 (d, 1H, =CHPh, $J=15.6$ $\mathrm{Hz}), 6.22\left(\mathrm{dt}, 1 \mathrm{H},=\mathrm{CHCH}_{2}, J=15.6 \mathrm{~Hz}, 7.8 \mathrm{~Hz}\right), 2.48\left(\mathrm{~s}, 2 \mathrm{H}, \mathrm{CH}_{2} \mathrm{~N}\right), 2.12(\mathrm{~d}, 2 \mathrm{H}$, $\mathrm{CH}_{2} \mathrm{CH}=, J=7.3 \mathrm{~Hz}$ ), 1.35 (br s, $2 \mathrm{H}, \mathrm{NH}_{2}$ ), 0.89 (s, $6 \mathrm{H}, 2 \mathrm{CH}_{3}$ ).

${ }^{13} \mathrm{C} \mathrm{NMR}\left(\mathrm{CDCl}_{3}, 500 \mathrm{MHz}\right): \delta$ ppm 139.5, 133.5, 130.1, 128.9, 127.3, 125.9, 54.5, 44.6, 37.0, 25.9 .

\section{$N$-Methylpent-4-en-1-amine (10e)}

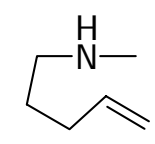

A solution of 5-bromopent-1-ene (5 g, $50 \mathrm{mmol})$ and methylamine $(15 \mathrm{~mL}, 400$ mmol) in diethyl ether $(15 \mathrm{~mL})$ was placed in a sealed tube and was heated at $30-40{ }^{\circ} \mathrm{C}$ for $40 \mathrm{~h}$ in a water bath. The lower boiling components were evaporated and the product was distilled at $106-109{ }^{\circ} \mathrm{C}$ to provide 10e, $3.2 \mathrm{~g}(64.5 \%){ }^{3 \mathrm{~b}}$

${ }^{1} \mathrm{H}$ NMR $\left(\mathrm{CDCl}_{3}, 500 \mathrm{MHz}\right): \delta$ ppm 5.73-5.79 (m, 1H, $\left.\mathrm{CH}=\mathrm{CH}_{2}\right), 4.88-4.98(\mathrm{~m}, 2 \mathrm{H}$, $\left.\mathrm{CH}=\mathrm{CH}_{2}\right), 2.51\left(\mathrm{t}, 2 \mathrm{H}, \mathrm{CH}_{2}, J_{\mathrm{HH}}=7 \mathrm{~Hz}\right), 2.36$ (s, 3H, NHMe), 2.01-2.05 (m, 2H, $\left.\mathrm{CH}_{2}\right)$, 1.5-1.55 (m, 2H, $\left.\mathrm{CH}_{2}\right)$.

${ }^{13} \mathrm{C} \mathrm{NMR}\left(\mathrm{CDCl}_{3}, 500 \mathrm{MHz}\right): \delta \mathrm{ppm} 139.5,114.9,52.0,37,31.9,29.5$. 


\section{Literature Cited}

[1] Y. Tamaru, M. Hoju, H. Higashumura, Z-I. Yoshida, J. Am. Chem. Soc. 1988, 110, 3994-4002.

[2] T. Kondo, T. Okada, T-a. Mitsudo, J. Am. Chem. Soc. 2002, 124, 186-187.

[3] a) R. A. Perry, S. C. Chen, B. C. Menon, K. Hanaya, Y. Chow, Can. J. Chem. 1976, 54, 2385-2401 ; b) M. Tokuda, Y. Yamada, T. Takagi, H. Suginome, A. Furusaki, Tetrahedron, 1987, 43, 281-296.

[4] E. M. Marlett, W. S. Park, J. Org. Chem. 1990, 55, 2968-2969.

[5] S. Vyskocil, S. Jaracz, M. Smrcina, M. Sticha, V. Hanus, M. Polasek, P. Kocovsky, J. Org. Chem. 1998, 63, 7727-7737. 
start point at $60 \mathrm{C}$
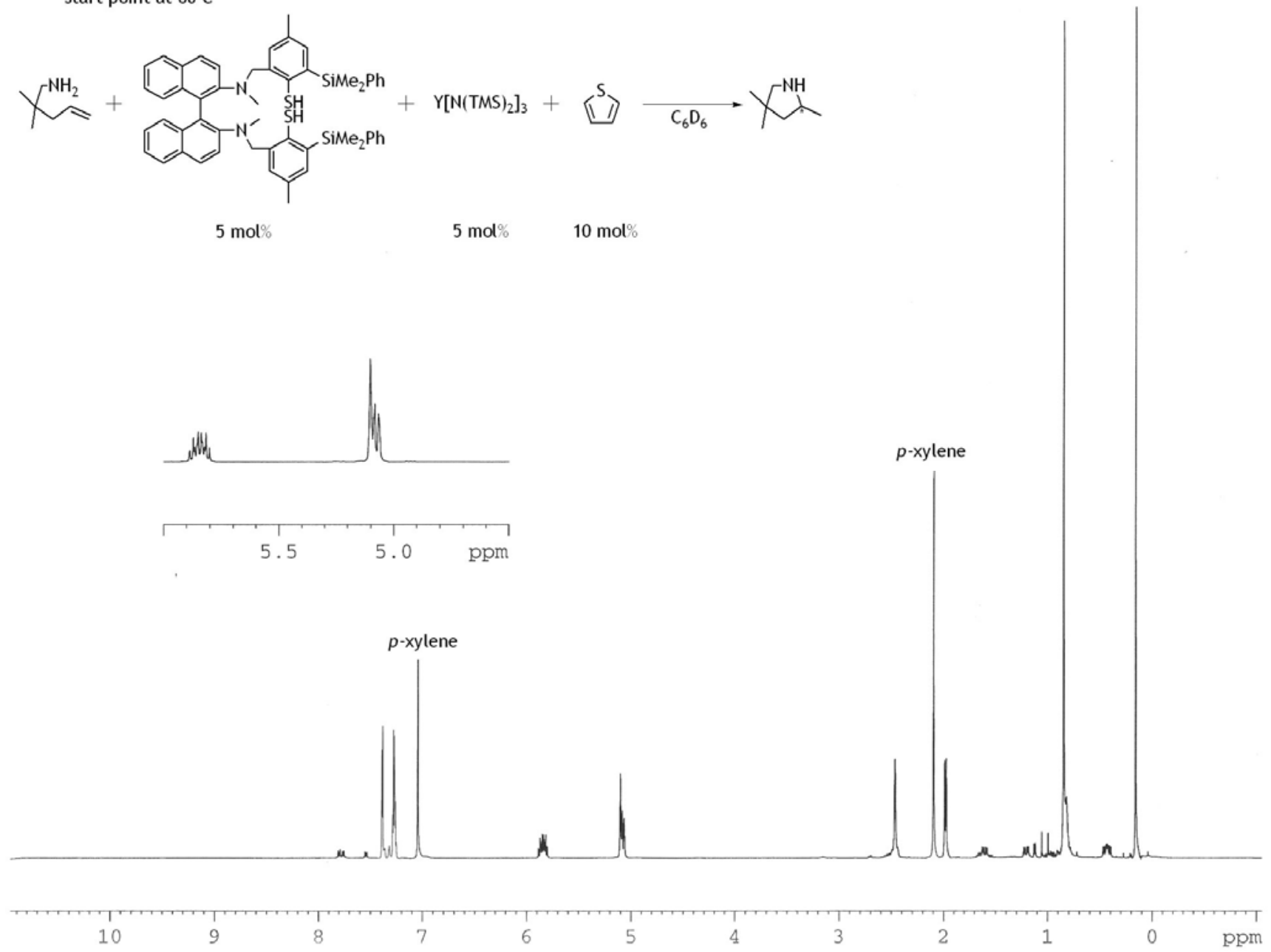

S16 


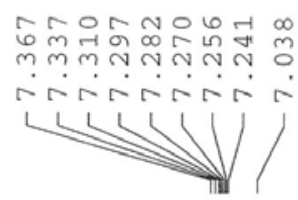

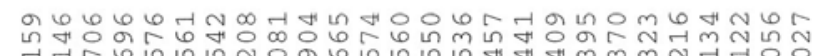
min่าivivin

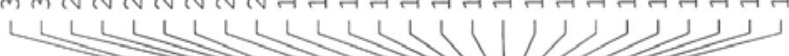
고 $\rightarrow$ m

$>95 \%$ conversion based on $p$-xylene after $60 \mathrm{C}, 9 \mathrm{~h}$

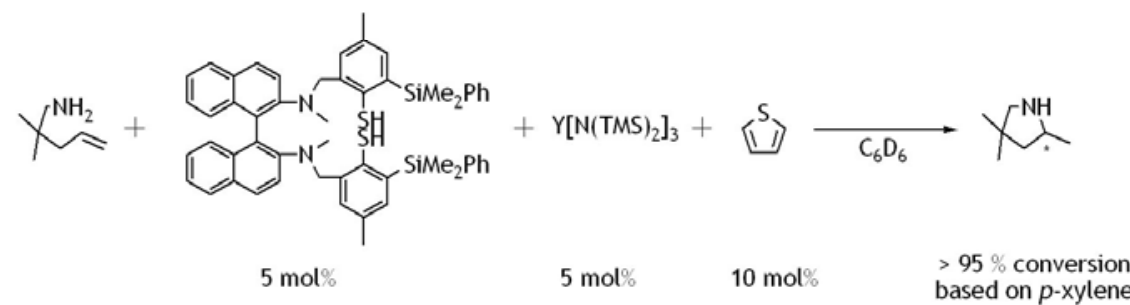

$p$-xylene

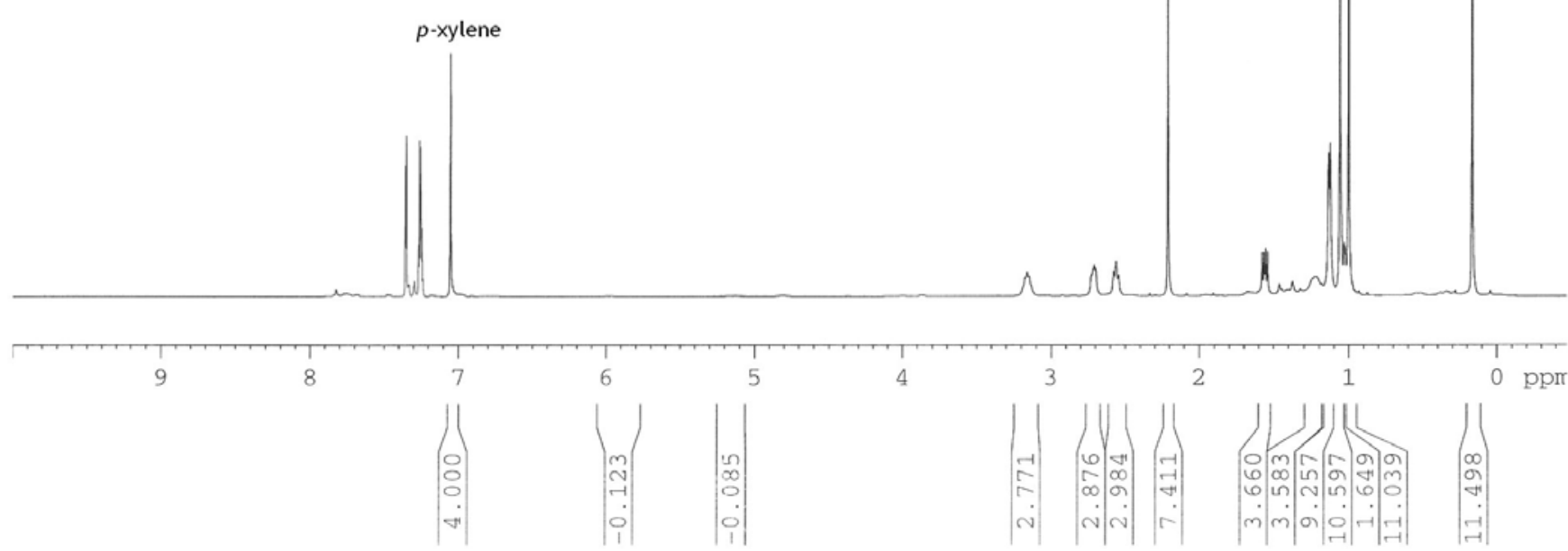




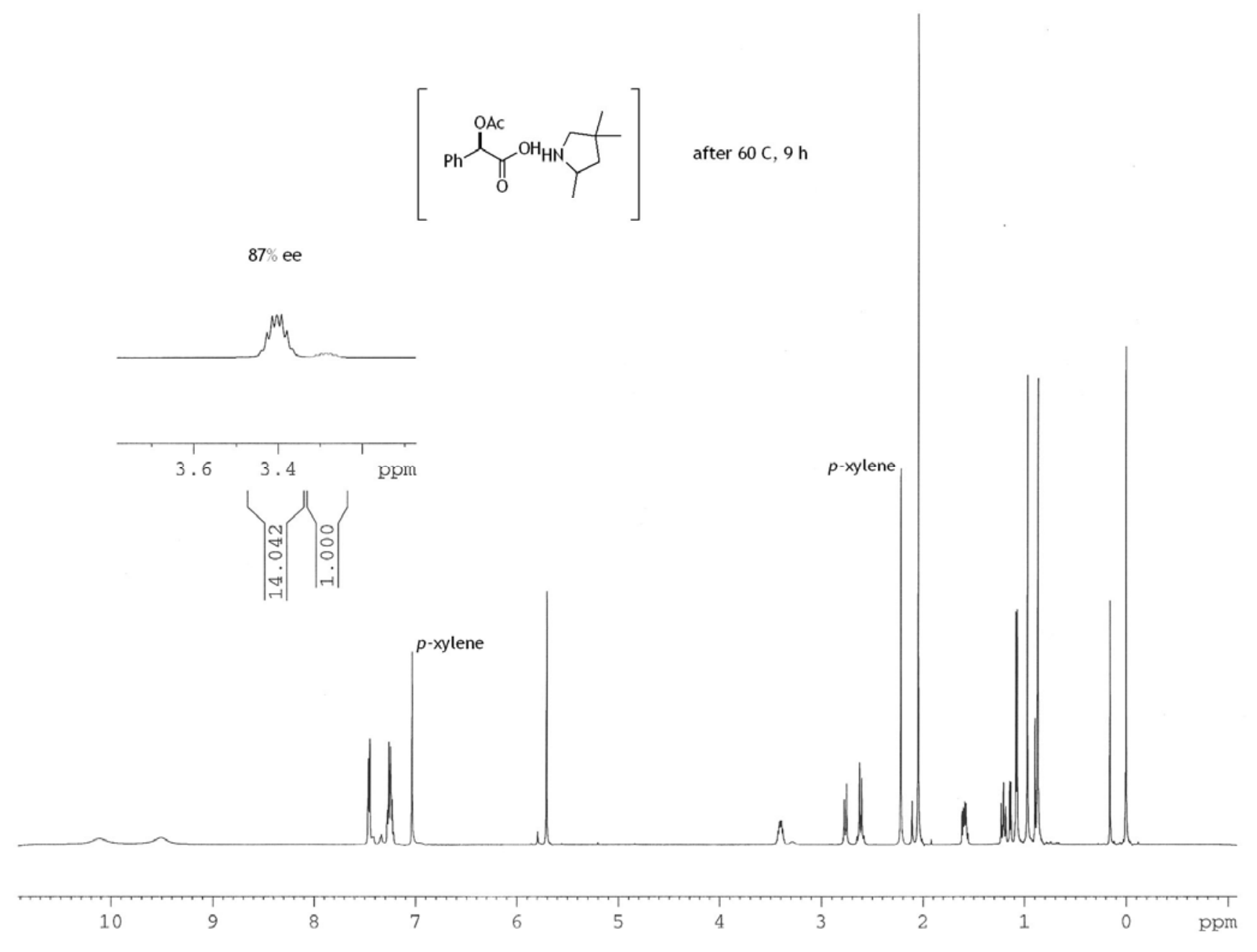




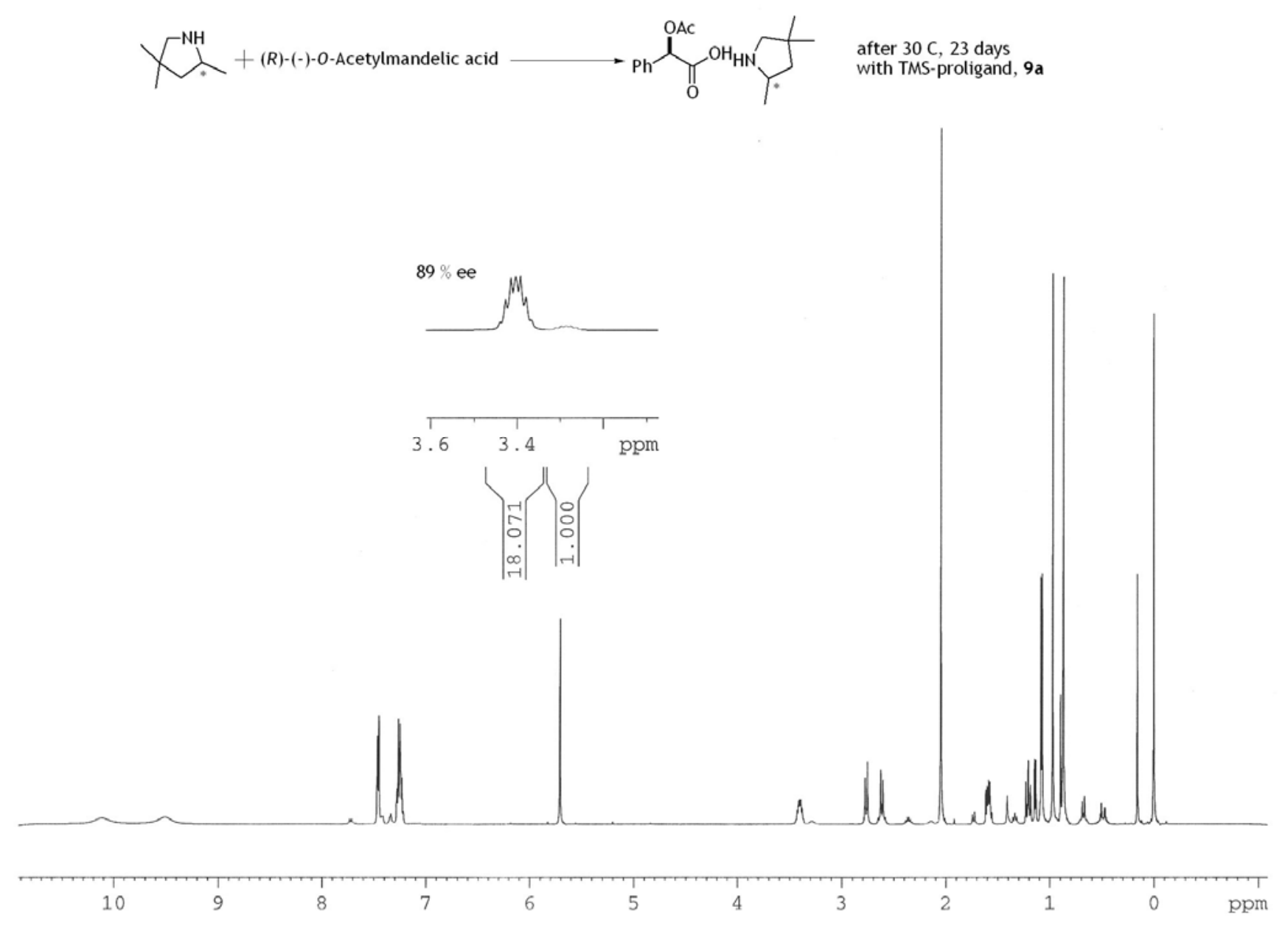




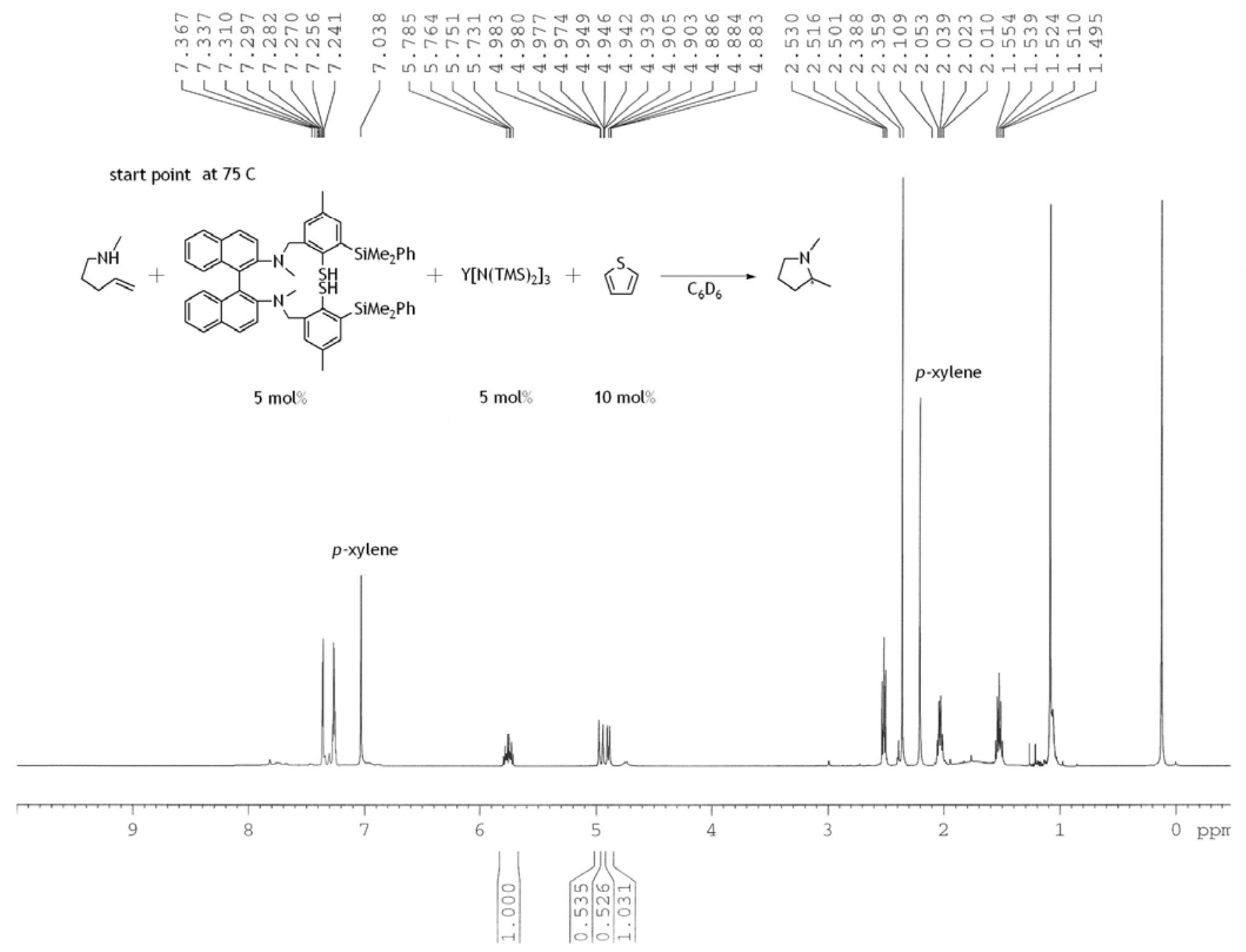




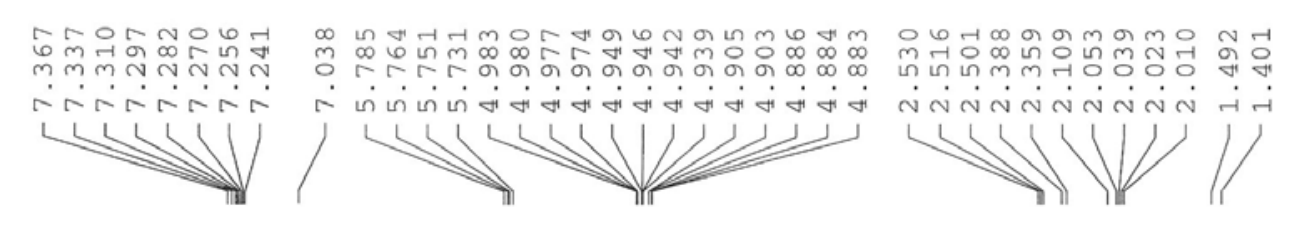

$>95 \%$ conversion based on $p$-xylene at $75 \mathrm{C}, 30 \mathrm{~h}$

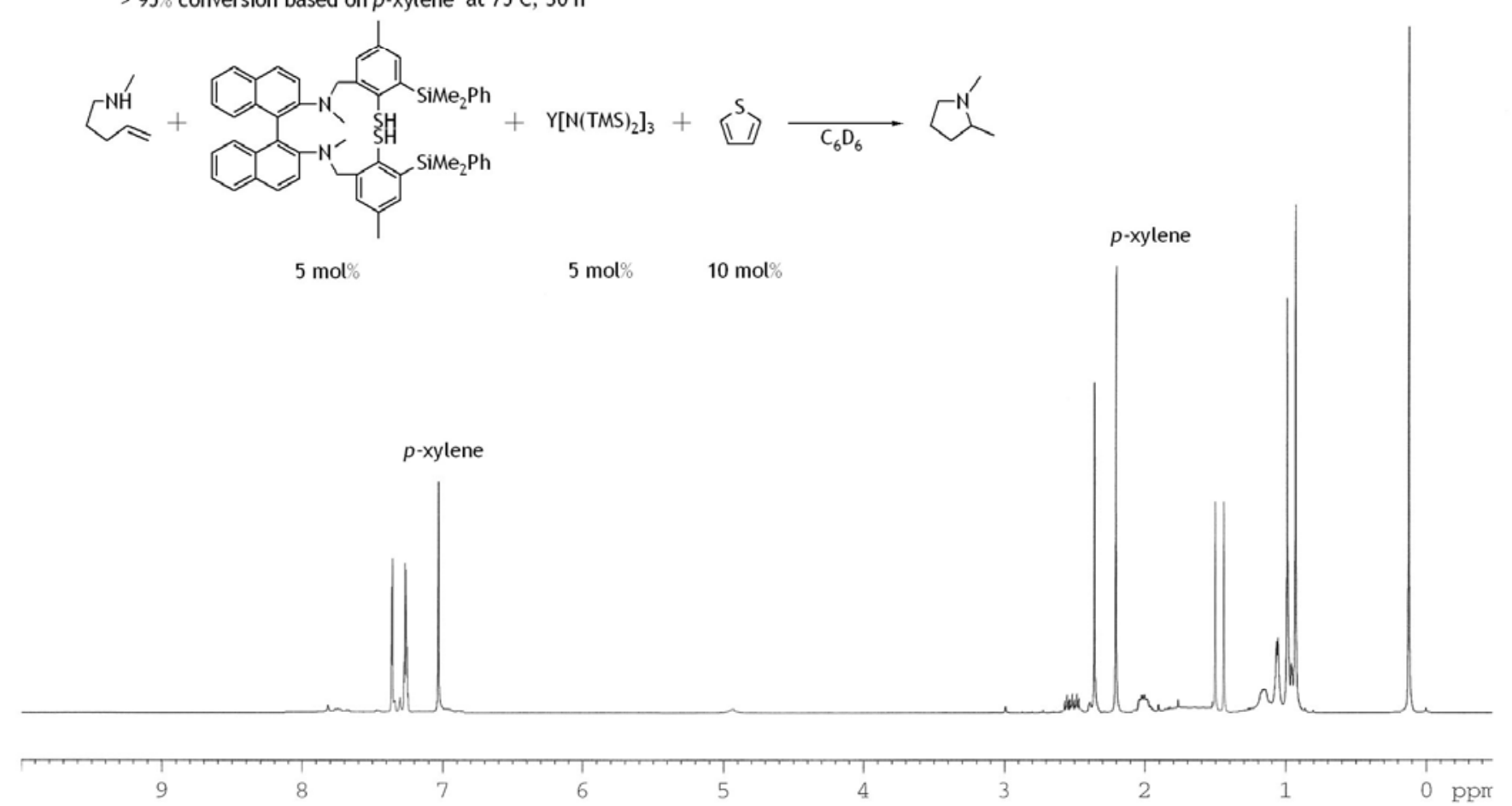




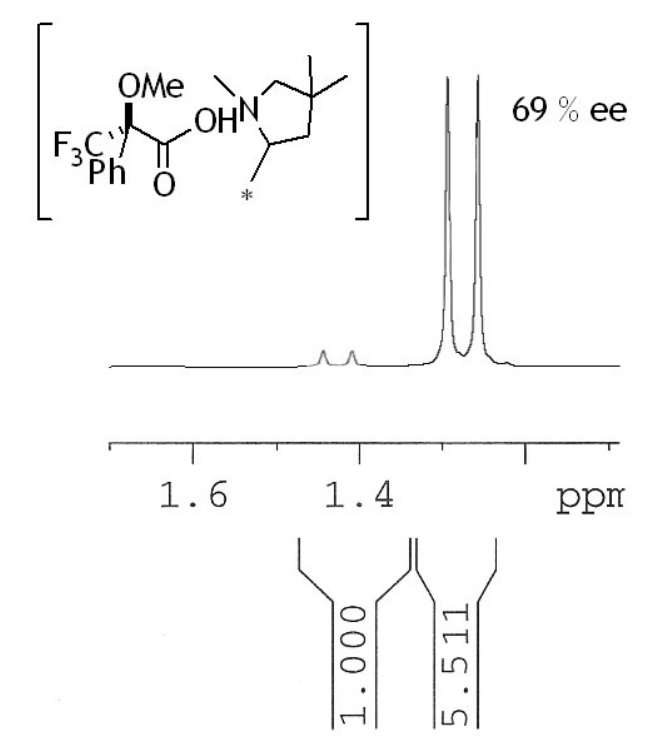

